\title{
THETA LIFTS AND LOCAL MAASS FORMS
}

\author{
Kathrin Bringmann, Ben Kane and Maryna Viazovska
}

\begin{abstract}
The first two authors and Kohnen have recently introduced a new class of modular objects called locally harmonic Maass forms, which are annihilated almost everywhere by the hyperbolic Laplacian operator. In this paper, we realize these locally harmonic Maass forms as theta lifts of harmonic weak Maass forms. Using the theory of theta lifts, we then construct examples of (non-harmonic) local Maass forms, which are instead eigenfunctions of the hyperbolic Laplacian almost everywhere.
\end{abstract}

\section{Introduction and statement of results}

In [7], a new class of modular objects was introduced. These functions, known as locally harmonic Maass forms, satisfy negative weight modularity and are annihilated almost everywhere by the hyperbolic Laplacian (see Section 2 for the relevant definitions), mirroring harmonic weak Maass forms. Recent interest in harmonic weak Maass forms began with their systematic treatment by Bruinier and Funke [13]. Following their appearance in the theory of mock theta functions due to Zwegers [40], it has been shown that harmonic weak Maass forms have applications ranging from partition theory (for example [2, 4, 6, 9, 11]) and Zagier's duality [39] relating "modular objects" of different weights (for example [10]) to derivatives of $L$-functions (for example $[14,15]$ ). They also arise in mathematical physics, as recently evidenced in Eguchi et al. [16] investigation of moonshine for the largest Mathieu group $M_{24}$. The main difference between locally harmonic Maass forms and harmonic weak Maass forms is that there are certain geodesics along which locally harmonic weak Maass forms are not necessarily real analytic and may even exhibit discontinuities.

In this paper, we realize the locally harmonic Maass forms studied in [7] as theta lifts of harmonic weak Maass forms. Theta lifts form connections between different types of modular objects. In particular, the Shimura lift [32] was realized as a theta lift by Niwa [25]. Niwa constructed this theta lift from an integral quadratic form of signature $(2,1)$, whereas Oda [26] extended this lift to quadratic forms of signature $(2, n)$ for $n \geq 1$. The theta lifts of interest in this paper may moreover be viewed in the framework of a much more general theta correspondence between automorphic forms associated to the two groups of a dual reductive pair [21]. Rallis and Schiffmann $[29,28,30,31]$ also constructed a lifting using the theta kernel furnished by the Weil representation. For example, Katok and Sarnak [22] used theta lifts to relate the central value of the $L$-series of a Maass cusp form to the Fourier coefficients of corresponding Maass cusp forms under the Shimura lift. This extended a famous

Received by the editors September 9, 2012.

2010 Mathematics Subject Classification. 11F37, 11F27, 11F11, 11F25, 11E16.

Key words and phrases. theta lifts, harmonic weak Maass forms, locally harmonic Maass forms, local Maass forms, modular forms. 
result of Waldspurger [36] proving that the central value of the $L$-function of an integral weight Hecke eigenform is proportional to the square of a coefficient of its half-integral weight counterpart under the Shintani lift. Tunnell [34] later exploited this link to express the central value of the $L$-function of an elliptic curve in terms of the coefficients of a theta function associated to a ternary quadratic form. Tunnell's Theorem gives a solution to the ancient congruent number problem (conditional on the Birch and Swinnerton-Dyer conjecture).

Although these classical lifts were restricted to cusp forms, the regularization of Harvey-Moore [19] and Borcherds [3] allows one to extend their definitions to previously divergent theta integrals. These extended theta lifts have more recently appeared in a variety of applications including generalized Kac-Moody algebras [18] and the arithmetic of Shimura varieties [15]. Following Bruinier's [12] application of Borcherds lifts to harmonic weak Maass forms, Bruinier and Funke [13] extended theta lifts to harmonic weak Maass forms. Due to the theory built around theta lifts, one may naturally extend the definition of locally harmonic Maass forms to include local Maass forms, i.e., functions with the above properties of locally harmonic Maass forms except that instead of being annihilated by the hyperbolic Laplacian, they are eigenfunctions. The locally harmonic Maass forms investigated in [7] have a natural connection to the Shimura [32] and Shintani [33] lifts, which we next describe.

For $k \in 2 \mathbb{N}$ and a discriminant $D>0$, Zagier [37] defined the functions

$$
f_{k, D}(z):=\frac{D^{k-\frac{1}{2}}}{\left(\begin{array}{c}
2 k-2 \\
k-1
\end{array}\right) \pi} \sum_{Q \in \mathcal{Q}_{D}} Q(z, 1)^{-k}
$$

where $\mathcal{Q}_{D}$ denotes the set of integral binary quadratic forms of discriminant $D \in \mathbb{Z}$ and for $Q=[a, b, c] \in \mathcal{Q}_{D}$ we have $Q(z, 1)=a z^{2}+b z+c$. Zagier showed that $f_{k, D} \in S_{2 k}$, the space of weight $2 k$ cusp forms for $\mathrm{SL}_{2}(\mathbb{Z})$ and it was later noticed that the $f_{k, D}$ could be naturally realized as (linear combinations of) hyperbolic Poincaré series defined by Petersson [27]. The functions $f_{k, D}$ reappeared in the (holomorphic) kernel function for the Shimura and Shintani lifts

$$
\Omega(z, \tau):=\sum_{0<D \equiv 0,1} f_{k, D}(z) e^{2 \pi i D \tau}
$$

between $S_{2 k}$ and $S_{k+\frac{1}{2}}^{+}$(Kohnen's plus space of weight $k+\frac{1}{2}$ modular forms), which was defined by Kohnen and Zagier [24]. For $g \in S_{k+\frac{1}{2}}^{+}$, the Petersson inner product $\langle g, \Omega(-\bar{z}, \cdot)\rangle$ equals $(-1)^{k / 2} 2^{2-3 k}$ times the Shimura lift of $g$. Kohnen and Zagier used $\Omega$ to explicitly compute the constant of proportionality in Waldspurger's result, in turn proving non-negativity of the central $L$-values of Hecke eigenforms.

As indicated above, the functions $f_{k, D}$ may be interpreted in terms of theta lifts. To describe this, we define Shintani's [33] non-holomorphic kernel function. Throughout we write $\tau=u+i v \in \mathbb{H}, z=x+i y \in \mathbb{H}$, and denote, for $Q=[a, b, c] \in \mathcal{Q}_{D}$,

$$
Q_{z}:=\frac{1}{y}\left(a|z|^{2}+b x+c\right)
$$


Using this notation, Shintani's theta function projected into Kohnen's plus space equals

$$
\Theta(z, \tau):=y^{-2 k} v^{\frac{1}{2}} \sum_{D \in \mathbb{Z}} \sum_{Q \in \mathcal{Q}_{D}} Q(z, 1)^{k} e^{-4 \pi Q_{z}^{2} v} e^{2 \pi i D \tau} .
$$

The function $\Theta(-\bar{z}, \tau)$ transforms like a modular form of weight $k+\frac{1}{2}$ in $\tau$ and weight $2 k$ in $z$ (see Proposition $3.2(1)$ ). Integrating the $D$ th weight $k+\frac{1}{2}$ (holomorphic) Poincaré series against $\Theta$ yields $f_{k, D}$.

One can use Borcherds's [3] regularized version $\langle f, g\rangle^{\text {reg }}$ of the Petersson inner product (see Section 2 for a definition) to extend the utility of the Shimura lift (realized as Niwa's [25] theta lift) to weak Maass forms. To be more precise, for a weight $k+\frac{1}{2}$ weak Maass form $H$ with eigenvalue

$$
\lambda_{s}:=\left(s-\frac{k}{2}-\frac{1}{4}\right)\left(1-s-\frac{k}{2}-\frac{1}{4}\right)
$$

under the hyperbolic Laplacian $\Delta_{k+\frac{1}{2}}$, we define the theta lift

$$
\Phi_{k}(H)(z):=\langle H, \Theta(z, \cdot)\rangle^{\mathrm{reg}} .
$$

By choosing an appropriate input, this lift leads to the natural generalization

$$
f_{k, s, D}(z):=\sum_{Q \in \mathcal{Q}_{D}} Q(z, 1)^{-k} \varphi_{s}\left(\frac{D y^{2}}{|Q(z, 1)|^{2}}\right)
$$

of $f_{k, D}$. Here, for $0<w \leq 1$ and $\operatorname{Re}(s) \geq \frac{k}{2}+\frac{1}{4}$, using the usual ${ }_{2} F_{1}$ notation for Gauss's hypergeometric function, we define

$$
\varphi_{s}(w):=\frac{\Gamma\left(s+\frac{k}{2}-\frac{1}{4}\right) D^{\frac{k}{2}+\frac{1}{4}}}{6 \Gamma(2 s)(4 \pi)^{\frac{k}{2}-\frac{1}{4}}} w^{s-\frac{k}{2}-\frac{1}{4}}{ }_{2} F_{1}\left(s+\frac{k}{2}-\frac{1}{4}, s-\frac{k}{2}-\frac{1}{4} ; 2 s ; w\right),
$$

which is easily seen to be a constant when $s=\frac{k}{2}+\frac{1}{4}$. Note that for $\operatorname{Re}(s)>\frac{k}{2}+\frac{1}{4}$, the Euler integral representation of the ${ }_{2} F_{1}$ (see (4.3)) yields

$$
\begin{aligned}
\varphi_{s}(w)= & \frac{\Gamma\left(s+\frac{k}{2}-\frac{1}{4}\right) D^{\frac{k}{2}+\frac{1}{4}}}{6 \Gamma\left(s+\frac{k}{2}+\frac{1}{4}\right) \Gamma\left(s-\frac{k}{2}-\frac{1}{4}\right)(4 \pi)^{\frac{k}{2}-\frac{1}{4}}} \\
& \times w^{s-\frac{k}{2}-\frac{1}{4}} \int_{0}^{1}(1-t)^{s+\frac{k}{2}-\frac{3}{4}} t^{s-\frac{k}{2}-\frac{5}{4}}(1-w t)^{-s-\frac{k}{2}+\frac{1}{4}} d t .
\end{aligned}
$$

In order to obtain the functions $f_{k, s, D}$, we apply the theta lift $\Phi_{k}$ to the $D$ th Poincaré series $P_{k+\frac{1}{2}, s, D}$ (see (2.12)) of weight $k+\frac{1}{2}$ with eigenvalue $\lambda_{s}$ under $\Delta_{k+\frac{1}{2}}$ in Kohnen's plus space. In the special case that $s=\frac{k}{2}+\frac{1}{4}$, this Poincaré series is precisely the classical cuspidal Poincaré series and $f_{k, \frac{k}{2}+\frac{1}{4}, D}$ is essentially $f_{k, D}$ because $\varphi_{\frac{k}{2}+\frac{1}{4}}$ is a constant.

We next show that in general the functions $f_{k, s, D}$ are local Maass forms with exceptional set given by the closed geodesics

$$
E_{D}:=\left\{z=x+i y \in \mathbb{H}: \exists a, b, c \in \mathbb{Z}, b^{2}-4 a c=D, a|z|^{2}+b x+c=0\right\} .
$$

Theorem 1.1. Suppose that $s \in \mathbb{C}$ satisfies $\operatorname{Re}(s) \geq \frac{k}{2}+\frac{1}{4}$ and $D>0$ is a discriminant. Then the following hold. 
(1) The function $f_{k, s, D}$ is a local Maass form of weight $2 k$ and eigenvalue $4 \lambda_{s}$ under $\Delta_{2 k}$ with exceptional set $E_{D}$. Moreover,

$$
f_{k, \frac{k}{2}+\frac{1}{4}, D}=\frac{2^{2 k-3}}{3(2 k-1)}(4 \pi D)^{\frac{3}{4}-\frac{k}{2}} f_{k, D},
$$

which is a cusp form.

(2) The theta lift $\Phi_{k}$ maps weight $k+\frac{1}{2}$ weak Maass forms with eigenvalue $\lambda_{s}$ under $\Delta_{k+\frac{1}{2}}$ to weight $2 k$ local Maass forms with eigenvalue $4 \lambda_{s}$ under $\Delta_{2 k}$. In particular, the image of the Dth Poincaré series under the theta lift $\Phi_{k}$ equals

$$
\Phi_{k}\left(P_{k+\frac{1}{2}, s, D}\right)=f_{k, s, D}
$$

Remark. The function $f_{k, s, D}$ is continuous for every $s \in \mathbb{C}$ with $\operatorname{Re}(s) \geq \frac{k}{2}+\frac{1}{4}$, but whenever $\lambda_{s} \neq 0$ there exist points along $E_{D}$ at which $f_{k, s, D}$ is not differentiable (for example, if $\tau \in S_{Q}$ for precisely two $Q \in \mathcal{Q}_{D}$ ). In particular, one should note the surprising fact that while the functions are not differentiable for $\lambda_{s} \neq 0$, the case $\lambda_{s}=0$ yields a (holomorphic) cusp form by (1.5).

We now investigate the general properties of the theta lift. Let $T_{p}$ and $T_{p^{2}}$ denote the Hecke operators of integral and half-integral weight, respectively (see (2.3) and (2.4)). We next show that the theta lift commutes with the Hecke operators.

Theorem 1.2. (1) For every weight $k+\frac{1}{2}$ weak Maass form $H$ with eigenvalue $\lambda_{s}$ with $\operatorname{Re}(s) \geq \frac{k}{2}+\frac{1}{4}$

$$
\left.\Phi_{k}(H)\right|_{2 k} T_{p}=\Phi_{k}\left(\left.H\right|_{k+\frac{1}{2}} T_{p^{2}}\right) .
$$

(2) If $\operatorname{Re}(s) \geq \frac{k}{2}+\frac{1}{4}$ and $s \neq \frac{k}{2}+\frac{1}{4}$, then the lift $\Phi_{k}$ is injective on the space of weak Maass forms with eigenvalue $\lambda_{s}$ under $\Delta_{k+\frac{1}{2}}$.

We next describe a theta lift that parallels the construction of Shintani [33] and Niwa [25] in negative weight. Define the following theta function:

$$
\Theta^{*}(z, \tau):=v^{k} \sum_{D \in \mathbb{Z}} \sum_{Q \in \mathcal{Q}_{D}} Q_{z} Q(z, 1)^{k-1} e^{-\frac{4 \pi|Q(z, 1)|^{2} v}{y^{2}}} e^{-2 \pi i D \tau} .
$$

The function $\Theta^{*}$ transforms like a modular form of weight $\frac{3}{2}-k$ in $\tau$ and weight $2-2 k$ in $z$ (see Proposition $3.2(2)$ ). Similar to the positive weight case, for a weak Maass form $H$ of weight $\frac{3}{2}-k$, we define the theta lift by

$$
\Phi_{1-k}^{*}(H)(z):=\left\langle H, \Theta^{*}(-\bar{z}, \cdot)\right\rangle^{\mathrm{reg}} .
$$

Since the space of weak Maass forms is spanned by the Poincaré series $P_{\frac{3}{2}-k, s, D}$ (defined in (2.12) ) [17], it suffices to consider their image under the theta lifting. This leads to the definition

$$
\mathcal{F}_{1-k, s, D}(z):=\sum_{Q \in \mathcal{Q}_{D}} \operatorname{sgn}\left(Q_{z}\right) Q(z, 1)^{k-1} \varphi_{s}^{*}\left(\frac{D y^{2}}{|Q(z, 1)|^{2}}\right),
$$


where, for $0<w \leq 1$ and $s \in \mathbb{C}$ with $\operatorname{Re}(s) \geq \frac{k}{2}-\frac{3}{4}$, we define

$$
\varphi_{s}^{*}(w):=\frac{\Gamma\left(s+\frac{k}{2}-\frac{1}{4}\right)(4 \pi D)^{\frac{3}{4}-\frac{k}{2}}}{12 \sqrt{\pi} \Gamma(2 s)} w^{\frac{k}{2}-\frac{3}{4}+s}{ }_{2} F_{1}\left(s-\frac{k}{2}+\frac{1}{4}, s+\frac{k}{2}-\frac{3}{4} ; 2 s ; w\right) .
$$

The Euler integral representation (4.3) again implies that

$$
\begin{aligned}
\varphi_{s}^{*}(w)= & \frac{\Gamma\left(s+\frac{k}{2}-\frac{1}{4}\right)(4 \pi D)^{\frac{3}{4}-\frac{k}{2}}}{12 \sqrt{\pi} \Gamma\left(s+\frac{k}{2}-\frac{3}{4}\right) \Gamma\left(s-\frac{k}{2}+\frac{3}{4}\right)} \\
& \times w^{\frac{k}{2}-\frac{3}{4}+s} \int_{0}^{1} t^{s+\frac{k}{2}-\frac{7}{4}}(1-t)^{s-\frac{k}{2}-\frac{1}{4}}(1-w t)^{-s+\frac{k}{2}-\frac{1}{4}} d t .
\end{aligned}
$$

In the special case that $s=\frac{k}{2}+\frac{1}{4}$, a change of variables yields the locally harmonic Maass form

$$
\mathcal{F}_{1-k, D}(z):=\frac{1}{12 \psi(1)}(4 \pi D)^{\frac{3}{4}-\frac{k}{2}} \sum_{Q \in \mathcal{Q}_{D}} \operatorname{sgn}\left(Q_{z}\right) Q(z, 1)^{k-1} \psi\left(\frac{D y^{2}}{|Q(z, 1)|^{2}}\right),
$$

investigated in [7]. Here

$$
\psi(v):=\frac{1}{2} \beta\left(v ; k-\frac{1}{2}, \frac{1}{2}\right)
$$

is a special value of the incomplete $\beta$-function, which is defined for $r, s \in \mathbb{C}$ satisfying $\operatorname{Re}(r), \operatorname{Re}(s)>0$ by $\beta(v ; s, r):=\int_{0}^{v} u^{s-1}(1-u)^{r-1} d u$. In [7], the first two authors and Kohnen introduced the functions $\mathcal{F}_{1-k, D}$ and showed that they transform like weight $2-2 k$ modular forms and are locally harmonic in every neighborhood of $\mathbb{H}$ which does not intersect $E_{D}$. More generally, the functions $\mathcal{F}_{1-k, s, D}$ are local Maass forms with exceptional set $E_{D}$, along which $\operatorname{sgn}\left(Q_{z}\right)=0$ causes discontinuities.

Theorem 1.3. Suppose that $k$ is even, $D>0$ is a discriminant, and $s \in \mathbb{C}$ satisfies $\operatorname{Re}(s) \geq \frac{k}{2}-\frac{3}{4}$. Then the following hold.

(1) The function $\mathcal{F}_{1-k, s, D}$ is a local Maass form of weight $2-2 k$ with eigenvalue $4 \lambda_{s}$ under $\Delta_{2-2 k}$ and exceptional set $E_{D}$.

(2) The theta lift $\Phi_{1-k}^{*}$ maps weight $\frac{3}{2}-k$ weak Maass forms with eigenvalue $\lambda_{s}$ under $\Delta_{\frac{3}{2}-k}$ to weight $2-2 k$ local Maass forms with eigenvalue $4 \lambda_{s}$ under $\Delta_{2-2 k}$. In particular, the image of $P_{\frac{3}{2}-k, s, D}$ under the theta lift is

$$
\Phi_{1-k}^{*}\left(P_{\frac{3}{2}-k, s, D}\right)=\mathcal{F}_{1-k, s, D}
$$

\section{Remarks.}

(1) The functions $\mathcal{F}_{1-k, s, D}$ are never continuous. That is to say, for every $s$ and $D$ satisfying the conditions of Theorem 1.3, there exist points along $E_{D}$ for which $\mathcal{F}_{1-k, s, D}$ exhibits discontinuities. For example, one can easily show that these occur at points where $\tau \in S_{Q}$ for precisely two $Q \in \mathcal{Q}_{D}$.

(2) Although $\mathcal{F}_{1-k, D}$ is never continuous, one may add a piecewise polynomial function to obtain a real analytic function. The polynomial in question is related to the period polynomial of $f_{k, D}$ and was thoroughly investigated in [7].

(3) In the omitted case $k=1$ and $\lambda_{s}=0$, Hövel [20] has constructed locally harmonic Maass forms via a theta lift. The relationship with the Shimura and Shintani lifts as well as its geometric interpretation were further investigated there. 
(4) The regularized theta lifts considered here should also have a geometric interpretation. One expects that their images represent cohomology classes of geodesic cycles as currents.

We again turn to the general properties of this theta lift. In particular, it also commutes with the Hecke operators.

Theorem 1.4. Suppose that $s \in \mathbb{C}$ satisfies $\operatorname{Re}(s) \geq \frac{k}{2}-\frac{3}{4}$. The following hold.

(1) For every weak Maass form $H$ of weight $\frac{3}{2}-k$ in Kohnen's plus space with eigenvalue $\lambda_{s}$ under $\Delta_{\frac{3}{2}-k}$, one has

$$
\left.\Phi_{1-k}^{*}(H)\right|_{2-2 k} T_{p}=\Phi_{1-k}^{*}\left(\left.H\right|_{\frac{3}{2}-k} T_{p^{2}}\right) .
$$

(2) The lift $\Phi_{1-k}^{*}$ is injective on the space of weak Maass forms with eigenvalue $\lambda_{s}$ under $\Delta_{\frac{3}{2}-k}$.

Remark. In [7], it was shown that the functions $\mathcal{F}_{1-k, D}$ satisfy relations under the Hecke operators which seemed to imply a natural connection to weight $\frac{3}{2}-k$ objects. This is explained by the relation (1.9) between integral and half-integral weight Hecke operators.

The images $\mathcal{F}_{1-k, s, D}$ and $f_{k, s, D}$ under the two theta lifts considered in this paper are related through the antiholomorphic differential operator $\xi_{\kappa}:=2 i y^{\kappa} \frac{\bar{\partial}}{\partial \bar{z}}$.

Theorem 1.5. Suppose that $k>0$ is an even integer, $D$ is a positive discriminant, and $s \in \mathbb{C}$ satisfies $\operatorname{Re}(s) \geq \frac{k}{2}+\frac{1}{4}$.

(1) For every $z \notin E_{D}$, we have that

$$
\xi_{2-2 k}\left(\mathcal{F}_{1-k, s, D}(z)\right)=2\left(\bar{s}-\frac{3}{4}+\frac{k}{2}\right) f_{k, \bar{s}, D}(z) .
$$

(2) For $z \notin E_{D}$, we have that

$$
\xi_{2 k}\left(f_{k, s, D}(z)\right)=2\left(\bar{s}-\frac{k}{2}-\frac{1}{4}\right) \mathcal{F}_{1-k, \bar{s}, D}(z) .
$$

Theorem 1.5 states that for $s \geq \frac{k}{2}+\frac{1}{4}$ the following commutative diagram holds:

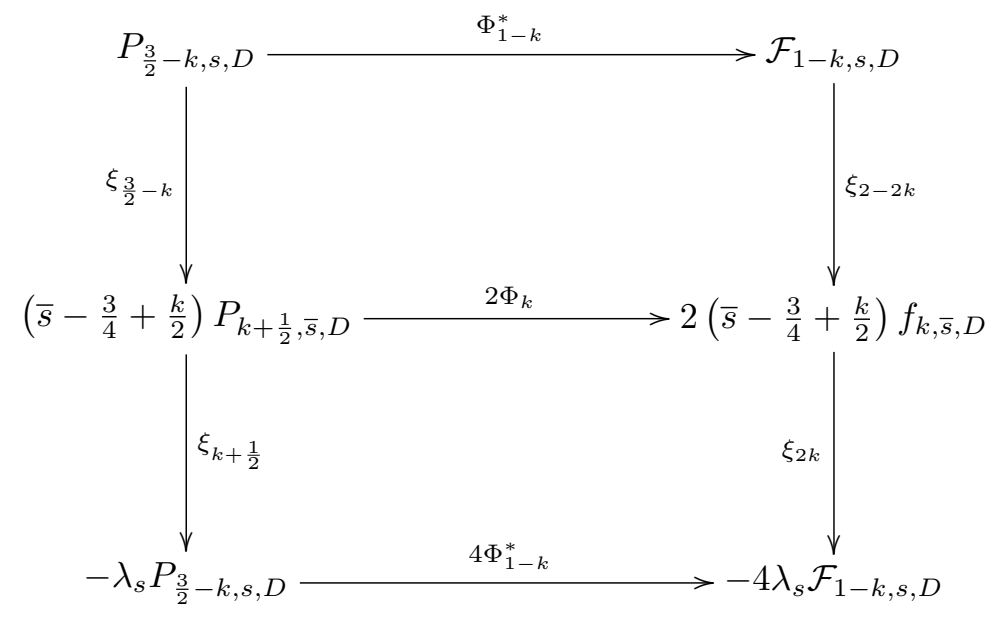


Denote the $d$ th Shimura [32] lift by $\mathscr{S}_{d}$ and $P_{\kappa, D}:=P_{\kappa, \frac{k}{2}+\frac{1}{4}, D}$. In the special case that $s=\frac{k}{2}+\frac{1}{4}$ (see Corollary 9 of [24] for the constant multiple of $\mathscr{S}_{1}$ ), the diagram becomes the following:

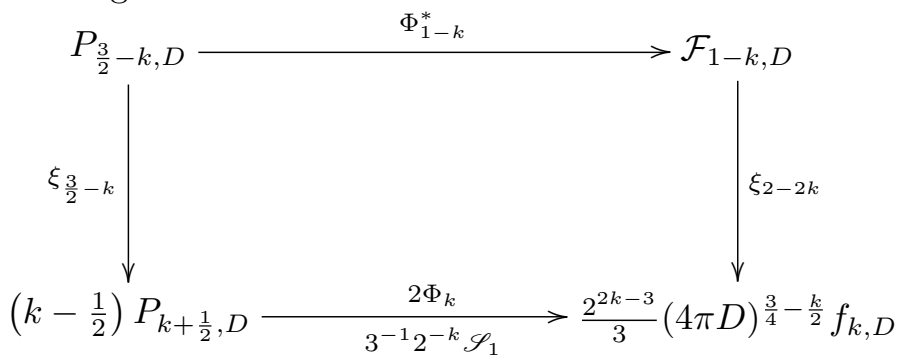

\section{Remarks.}

(1) The above diagram extends work of Bruinier and Funke [13] and Hövel [20] in the case of $O(2,1)$ to higher weight.

(2) By applying (6.1) (used to obtain (1.10)) to $s$-derivatives of weak Maass forms, one could also obtain links between modular objects known as sesquiharmonic forms [5]. These functions map to weakly holomorphic modular forms under the hyperbolic Laplacian.

The paper is organized as follows. In Section 2, we recall the theory of weak Maass forms and give a formal definition of local Maass forms. Section 2.1 is devoted to the properties of the regularized inner product. The modularity properties of the theta functions are described in Section 3, where we derive a number of interrelations between the theta functions through differential operators. The image of $\Phi_{k}$ (Theorem $1.1(2))$ is determined in Section 4, while Section 5 is devoted to the image of $\Phi_{1-k}^{*}$ (Theorem $1.3(2)$ ) and the injectivity of the lift (Theorem $1.4(2)$ ). In Section 6, Theorem 1.5 is established and the relationship between $f_{k, s, D}$ and $\mathcal{F}_{1-k, s, D}$ is then used to conclude Theorems 1.1 (1) and 1.3 (1). Finally, Section 7 concludes the paper with a discussion of the Hecke operators and the injectivity of $\Phi_{k}$ (Theorems 1.2 and $1.4(1))$.

\section{Basic facts on weak and local Maass forms}

In this section, we recall the basic definitions necessary to describe the modular objects and the theta lifts used in this paper. We first define the regularized inner product used in the definitions of $\Phi$ and $\Phi^{*}$. In order to understand the relationship between lifts in different spaces, we then define the Hecke operators, which act formally on any translation invariant function. We then recall Kohnen's plus space and weak Maass forms, to which we apply our theta lifts. The next subsection is devoted to constructing Poincaré series which span these spaces of weak Maass forms. Following this, we give the definition of local Maass forms, which are the focus of this paper.

Thoughout this section, $\kappa \in \frac{1}{2} \mathbb{Z}$ and we set $\Gamma:=\mathrm{SL}_{2}(\mathbb{Z})$ whenever $\kappa \in \mathbb{Z}$, while $\Gamma:=\Gamma_{0}(4)$ if $\kappa \in \frac{1}{2} \mathbb{Z} \backslash \mathbb{Z}$.

2.1. Regularized inner products and Hecke operators. For $T>0$, denote the truncated fundamental domain for $\mathrm{SL}_{2}(\mathbb{Z})$ by

$$
\mathcal{F}_{T}:=\left\{\tau \in \mathbb{H}:|u| \leq \frac{1}{2},|\tau| \geq 1, v \leq T\right\} .
$$


For a finite index subgroup $\Gamma \subseteq \mathrm{SL}_{2}(\mathbb{Z})$, we choose a set of representatives of $\Gamma \backslash \mathrm{SL}_{2}(Z)$ and further define

$$
\mathcal{F}_{T}(\Gamma):=\bigcup_{\gamma \in \Gamma \backslash \mathrm{SL}_{2}(\mathbb{Z})} \gamma \mathcal{F}_{T}
$$

In particular, we set $\mathcal{F}_{T}(4):=\mathcal{F}_{T}\left(\Gamma_{0}(4)\right)$. For two functions $G$ and $H$ satisfying weight $\kappa$ modularity for the group $\Gamma$, we define, whenever the limit exists, the regularized inner product

$$
\langle G, H\rangle^{\mathrm{reg}}:=\frac{1}{\left[\mathrm{SL}_{2}(\mathbb{Z}): \Gamma\right]} \lim _{T \rightarrow \infty} \int_{\mathcal{F}_{T}(\Gamma)} G(\tau) \overline{H(\tau)} v^{\kappa} \frac{d u d v}{v^{2}} .
$$

Although $\mathcal{F}_{T}$ depends on a set of representatives of $\Gamma \backslash \mathrm{SL}_{2}(\mathbb{Z})$, the inner product $\langle G, H\rangle^{\text {reg }}$ is independent of this choice. We often evaluate the inner product between $\xi_{\kappa}(G)$ and $H$, where $G$ satisfies weight $\kappa$ modularity, $H$ satisfies weight $2-\kappa$ modularity, and $\xi_{\kappa}=2 i y^{\kappa} \frac{\bar{\partial}}{\partial \bar{z}}$. For this, we use the following lemma, which follows by standard arguments using Stokes's Theorem.

Lemma 2.1. Suppose that $F, G: \mathbb{H} \rightarrow \mathbb{C}$ are real analytic functions that satisfy $\left.F\right|_{2-\kappa} \gamma=F$ and $\left.G\right|_{\kappa} \gamma=G$ for all $\gamma \in \Gamma$. Then

$$
\begin{aligned}
& \int_{\mathcal{F}_{T}(\Gamma)} \xi_{2-\kappa}(F(\tau)) \overline{G(\tau)} v^{\kappa-2} d u d v+\int_{\mathcal{F}_{T}(\Gamma)} \xi_{\kappa}(G(\tau)) \overline{F(\tau)} v^{-\kappa} d u d v \\
& \quad=-\int_{\partial \mathcal{F}_{T}(\Gamma)} \overline{F(\tau) G(\tau)} d \bar{\tau}
\end{aligned}
$$

A number of important operators are Hermitian with respect to the regularized inner product. One such class of operators is the Hecke operators. Suppose that $F$ is a function satisfying weight $\kappa$ modularity and write its Fourier expansion as

$$
F(\tau)=\sum_{n \in \mathbb{Z}} a_{v}(n) e^{2 \pi i n u}
$$

If $\kappa \in \mathbb{Z}$ (resp. $\kappa \in \frac{1}{2} \mathbb{Z} \backslash \mathbb{Z}$ ), then for a prime $p$, the Hecke operator $T_{p}$ (resp. $T_{p^{2}}$ ) is defined by

$$
\left.F\right|_{\kappa} T_{p}(\tau):=\sum_{n \in \mathbb{Z}}\left(a_{v}(p n)+p^{\kappa-1} a_{v}\left(\frac{n}{p}\right)\right) e^{2 \pi i n u}
$$

$$
\left.F\right|_{\kappa} T_{p^{2}}(\tau):=\sum_{n \in \mathbb{Z}}\left(a_{v}\left(p^{2} n\right)+p^{\kappa-\frac{3}{2}}\left(\frac{(-1)^{\kappa-\frac{1}{2}} n}{p}\right) a_{v}(n)+p^{2 \kappa-2} a_{v}\left(\frac{n}{p^{2}}\right)\right) e^{2 \pi i n u} .
$$

We apply the regularized inner product to (half-integral weight) weak Maass forms, which we define in the following subsection.

2.2. Weak Maass forms. When $\kappa \in \frac{1}{2} \mathbb{Z} \backslash \mathbb{Z}$, we are interested in weight $\kappa$ real analytic modular forms on $\Gamma$ in Kohnen's plus space. This means that the Fourier expansions are supported on the coefficients $n$ satisfying $(-1)^{\kappa-\frac{1}{2}} n \equiv 0,1(\bmod 4)$. We use pr to denote the projection operator (see Section 2.3 of [23]) into Kohnen's plus space. It is useful to recall that if $F$ is modular in Kohnen's plus space for $\Gamma$, 
then its Fourier expansions at the cusps 0 and $\frac{1}{2}$ are determined by the expansion at $i \infty$ (see [23] for a proof in the holomorphic case). Like the Hecke operators, the projection operator pr is Hermitian with respect to the regularized inner product, i.e.,

$$
\langle G \mid \operatorname{pr}, H\rangle^{\text {reg }}=\langle G, H \mid \operatorname{pr}\rangle^{\text {reg }} .
$$

The real analytic modular forms of particular interest for this paper are weak Maass forms. A good background reference for weak Maass forms is [13]. Recall that we write $\tau=u+i v$ throughout. For $\kappa \in \frac{1}{2} \mathbb{Z}$, the weight $\kappa$ hyperbolic Laplacian is defined by

$$
\Delta_{\kappa}:=\Delta_{\kappa, \tau}:=-v^{2}\left(\frac{\partial^{2}}{\partial u^{2}}+\frac{\partial^{2}}{\partial v^{2}}\right)+i \kappa v\left(\frac{\partial}{\partial u}+i \frac{\partial}{\partial v}\right) .
$$

It is related to the operator $\xi_{\kappa}=\xi_{\kappa, \tau}:=2 i v^{\kappa} \overline{\frac{\partial}{\partial \bar{\tau}}}$ through

$$
\Delta_{\kappa}=-\xi_{2-\kappa} \circ \xi_{\kappa}
$$

In order to define weak Maass forms, we require

$$
\mathcal{M}_{\kappa, s}(t):=|t|^{-\frac{\kappa}{2}} M_{\frac{\kappa}{2} \operatorname{sgn}(t), s-\frac{1}{2}}(|t|),
$$

where $M_{\mu, s-\frac{1}{2}}$ is the usual $M$-Whittaker function. For $\operatorname{Re}(s \pm \mu)>0$ and $v>0$, we have the integral representation

$$
M_{\mu, s-\frac{1}{2}}(v)=v^{s} e^{\frac{v}{2}} \frac{\Gamma(2 s)}{\Gamma(s+\mu) \Gamma(s-\mu)} \int_{0}^{1} t^{s+\mu-1}(1-t)^{s-\mu-1} e^{-v t} d t
$$

In the special case that $\mu=s$, we have

$$
M_{\mu, s-\frac{1}{2}}(v)=e^{-\frac{v}{2}} v^{s} .
$$

Furthermore, as $v \rightarrow \infty$, the Whittaker function satisfies the following asymptotic behavior for $\mu \neq s$ :

$$
M_{\mu, s-\frac{1}{2}}(v) \sim \frac{\Gamma(2 s)}{\Gamma(s-\mu)} e^{\frac{v}{2}} v^{-\mu}
$$

We move on to the definition of weak Maass forms. For $s \in \mathbb{C}$ a weak Maass form $F: \mathbb{H} \rightarrow \mathbb{C}$ of weight $\kappa$ for $\Gamma$ with eigenvalue $\lambda=\left(s-\frac{\kappa}{2}\right)\left(1-s-\frac{\kappa}{2}\right)$ is a real analytic function satisfying:

(1) For every $\gamma \in \Gamma$, one has $\left.F\right|_{\kappa} \gamma=F$, where $\left.\right|_{\kappa}$ denotes the usual weight $\kappa$ slash-operator.

(2) One has $\Delta_{\kappa}(F)=\lambda F$.

(3) As $v \rightarrow \infty$, there exist $a_{1}, \ldots, a_{N} \in \mathbb{C}$ for which

$$
F(\tau)-\sum_{m=1}^{N} a_{m} \mathcal{M}_{\kappa, s}(4 \pi \operatorname{sgn}(\kappa) m v) e^{2 \pi i m \operatorname{sgn}(\kappa) u}=O\left(v^{1-\operatorname{Re}(s)-\frac{\kappa}{2}}\right) .
$$

There are analogous conditions at the other cusps of $\Gamma$. 
2.3. Poincaré series. One builds explicit examples of weak Maass forms by constructing Poincaré series [17]. For $m \in \mathbb{Z} \backslash\{0\}$, the function

$$
\psi_{m, \kappa}(s ; \tau):=(4 \pi|m|)^{\frac{\kappa}{2}} \Gamma(2 s)^{-1} \mathcal{M}_{\kappa, s}(4 \pi m v) e^{2 \pi i m u}
$$

is an eigenfunction for $\Delta_{\kappa}$ with eigenvalue $\left(s-\frac{\kappa}{2}\right)\left(1-s-\frac{\kappa}{2}\right)$. Thus, one concludes that for $\operatorname{Re}(s)>1$ the Poincaré series

$$
P_{\kappa, s, \Gamma, m}(\tau):=\left.\sum_{\gamma \in \Gamma_{\infty} \backslash \Gamma} \psi_{\operatorname{sgn}(\kappa) m, \kappa}(s ; \tau)\right|_{\kappa} \gamma,
$$

where $\Gamma_{\infty}:=\left\{ \pm\left(\begin{array}{cc}1 & n \\ 0 & 1\end{array}\right): n \in \mathbb{Z}\right\}$, is also an eigenfunction under $\Delta_{\kappa}$ with the same eigenvalue. Moreover, the space of weight $\kappa$ weak Maass forms with this eigenvalue is spanned by such Poincaré series. The Poincaré series satisfies the growth condition

$$
P_{\kappa, s, \Gamma, m}(\tau)-\psi_{\operatorname{sgn}(\kappa) m, \kappa}(s ; \tau)=O\left(v^{1-\operatorname{Re}(s)-\frac{\kappa}{2}}\right) .
$$

In the case that $\kappa \in \frac{1}{2} \mathbb{Z} \backslash \mathbb{Z}$, we then project the Poincaré series into Kohnen's plus space, defining

$$
P_{\kappa, s, m}:=P_{\kappa, s, \Gamma_{0}(4), m} \mid \operatorname{pr} .
$$

In the special cases that $s=1-\frac{\kappa}{2}$ or $s=\frac{\kappa}{2}$, the resulting Poincaré series is harmonic. For $D \neq 0$, the positive and negative weight Poincaré series are related to each other via

$$
\xi_{\kappa}\left(P_{\kappa, s, D}\right)=\left(\bar{s}-\frac{\kappa}{2}\right) P_{2-\kappa, \bar{s}, D}
$$

2.4. Local Maass forms. Mirroring the definition of weak Maass forms, for $\kappa \in 2 \mathbb{Z}$, $\lambda \in \mathbb{C}$, and a measure zero set $E$, we call a function $\mathcal{F}$ a weight $\kappa$ local Maass form with eigenvalue $\lambda$ and exceptional set $E$ if $\mathcal{F}$ satisfies the following:

(1) For every $\gamma \in \mathrm{SL}_{2}(\mathbb{Z})$, one has $\left.\mathcal{F}\right|_{\kappa} \gamma=\mathcal{F}$.

(2) For every $\tau \notin E$ there exists a neighborhood around $\tau$ for which $\mathcal{F}$ is real analytic and

$$
\Delta_{\kappa}(\mathcal{F})(\tau)=\lambda \mathcal{F}(\tau)
$$

(3) For $\tau \in E$ one has

$$
\mathcal{F}(\tau)=\frac{1}{2} \lim _{r \rightarrow 0^{+}}(\mathcal{F}(\tau+i r)+\mathcal{F}(\tau-i r)) .
$$

(4) As a function of $v, \mathcal{F}$ exhibits at most polynomial growth as $v \rightarrow \infty$.

Since $E_{D}$ is invariant under $\mathrm{SL}_{2}(\mathbb{Z})$ and $\mathcal{F}$ may exhibit discontinuities, we note that there would be no relationship between the values of $\mathcal{F}$ on $E_{D}$ and the values of $\mathcal{F}$ on $\mathbb{H} \backslash E_{D}$ without condition (3). Examples of locally harmonic Maass forms (those with eigenvalue 0) are given in [7] as "quadratic form Poincaré series." These functions satisfy condition (3) and serve as the source of its definition here. In this paper, we give further examples of local Maass forms via theta lifts. 


\section{Indefinite theta functions}

In this section, we collect several important properties of the theta functions (1.2) and (1.6). The modularity properties of these indefinite theta functions follow by a result of Vignéras [35]. To state these, we define the Euler operator $E:=\sum_{i=1}^{n} w_{i} \frac{\partial}{\partial w_{i}}$. As usual, we denote the Gram matrix associated to a non-degenerate quadratic form $q$ on $\mathbb{R}^{n}$ by $A$. The Laplacian associated to $q$ is then defined by $\Delta:=\left\langle\frac{\partial}{\partial w}, A^{-1} \frac{\partial}{\partial w}\right\rangle$. Here $\langle\cdot, \cdot\rangle$ denotes the usual inner product on $\mathbb{R}^{n}$.

Theorem 3.1 (Vignéras). Suppose that $n \in \mathbb{N}, q$ is a non-degenerate quadratic form on $\mathbb{R}^{n}, L \subset \mathbb{R}^{n}$ is a lattice on which $q$ takes integer values, and $p: \mathbb{R}^{n} \rightarrow \mathbb{C}$ is a function satisfying the following conditions:

(i) The function $f(w):=p(w) e^{-2 \pi q(w)}$ times any polynomial of degree at most 2 and all partial derivatives of $f$ of order at most 2 are elements of $L^{2}\left(\mathbb{R}^{n}\right) \cap$ $L^{1}\left(\mathbb{R}^{n}\right)$.

(ii) For some $\lambda \in \mathbb{Z}$, the function $p$ satisfies

$$
\left(E-\frac{\Delta}{4 \pi}\right) p=\lambda p
$$

Then the indefinite theta function

$$
v^{-\frac{\lambda}{2}} \sum_{w \in L} p(w \sqrt{v}) e^{2 \pi i q(w) \tau}
$$

is modular of weight $\lambda+\frac{n}{2}$ for $\Gamma_{0}(N)$ and character $\chi \cdot \chi_{-4}^{\lambda}$, where $N$ and $\chi$ are the level and character of $q$ and $\chi_{-4}$ is the unique primitive Dirichlet character of conductor 4.

Remark. Note that the definition of the character given in Vignéras [35] differs to that given by Shimura [32] by a factor of $\chi_{-4}^{\lambda}$. We adopt Shimura's notation here.

Applying Theorem 3.1 to $\Theta$ and $\Theta^{*}$ yields their modularity properties (see [8] for details).

\section{Proposition 3.2.}

(1) The function $\Theta(-\bar{z}, \tau)$ transforms like a modular form of weight $k+\frac{1}{2}$ in Kohnen's plus space on $\Gamma_{0}(4)$ in $\tau$ and weight $2 k$ on $\mathrm{SL}_{2}(\mathbb{Z})$ in $z$.

(2) The function $\Theta^{*}$ transforms like a modular form of weight $\frac{3}{2}-k$ in Kohnen's plus space on $\Gamma_{0}(4)$ in $\tau$ and weight $2-2 k$ on $\mathrm{SL}_{2}(\mathbb{Z})$ in $z$.

The following lemma is the key relation needed to establish a link between the functions $f_{k, s, D}$ and $\mathcal{F}_{1-k, s, D}$. The correspondence is formed through a relation between the respective differential operators in $\tau$ and $z$ on $\Theta$ and $\Theta^{*}$, mirroring an important connection formed in [13].

Lemma 3.3. For every integer $k \geq 1$, one has

$$
\begin{aligned}
\xi_{k+\frac{1}{2}, \tau}(\Theta(z, \tau)) & =-i y^{2-2 k} \frac{\partial}{\partial z} \Theta^{*}(-\bar{z}, \tau), \\
\xi_{\frac{3}{2}-k, \tau}\left(\Theta^{*}(-\bar{z}, \tau)\right) & =-i y^{2 k} \frac{\partial}{\partial z} \Theta(z, \tau) .
\end{aligned}
$$


Proof. We first prove (3.1). We compute that $\frac{\partial}{\partial z} \Theta^{*}(-\bar{z}, \tau)$ equals

$$
\sum_{\substack{D \in \mathbb{Z} \\ Q \in \mathcal{Q}_{D}}} Q(-\bar{z}, 1)^{k-1} e^{-\frac{4 \pi v}{y^{2}}|Q(-\bar{z}, 1)|^{2}} e^{-2 \pi i D \tau}\left(\frac{\partial}{\partial z} Q_{-\bar{z}}-4 \pi Q_{-\bar{z}} v \frac{\partial}{\partial z}\left(\frac{|Q(-\bar{z}, 1)|^{2}}{y^{2}}\right)\right) .
$$

We then use

$$
|Q(z, 1)|^{2}=Q_{z}^{2} y^{2}+D y^{2}
$$

and

$$
y^{2} \frac{\partial}{\partial z} Q_{-\bar{z}}=\frac{i}{2} Q(-\bar{z}, 1)
$$

to obtain

$$
-i y^{2-2 k} \frac{\partial}{\partial z} \Theta^{*}(-\bar{z}, \tau)=\frac{1}{2} y^{-2 k} v^{k} \sum_{\substack{D \in \mathbb{Z} \\ Q \in \mathcal{Q}_{D}}} Q(-\bar{z}, 1)^{k} e^{-4 \pi Q_{-\bar{z}}^{2} v} e^{-2 \pi i D \bar{\tau}}\left(1-8 \pi Q_{-\bar{z}}^{2} v\right) .
$$

We similarly compute the action of $\xi_{k+\frac{1}{2}, \tau}$ on $\Theta$. A straightforward calculation yields

$$
\xi_{k+\frac{1}{2}, \tau}(\Theta(z, \tau))=\frac{1}{2} y^{-2 k} v^{k} \sum_{\substack{D \in \mathbb{Z} \\ Q \in \mathcal{Q}_{D}}} Q(\bar{z}, 1)^{k} e^{-4 \pi Q_{z}^{2} v} e^{-2 \pi i D \bar{\tau}}\left(1-8 \pi Q_{z}^{2} v\right) .
$$

Equation (3.1) now follows immediately by the change of variables $Q=[a, b, c] \rightarrow$ $[a,-b, c]=: \widetilde{Q} \in \mathcal{Q}_{D}$, noting that

$$
\widetilde{Q}(\bar{z}, 1)=Q(-\bar{z}, 1) \text { and } \widetilde{Q}_{z}=Q_{-\bar{z}} .
$$

Next, we prove (3.2). Since $Q_{z} \in \mathbb{R}$, a direct calculation, mirroring the proof of (3.1) and using (3.4), yields

$$
\xi_{\frac{3}{2}-k, \tau}\left(\Theta^{*}(-\bar{z}, \tau)\right)=v^{\frac{1}{2}} \sum_{\substack{D \in \mathbb{Z} \\ Q \in \mathcal{Q}_{D}}} Q_{z} Q(z, 1)^{k-1} e^{-4 \pi Q_{z}^{2} v} e^{2 \pi i D \tau}\left(k-\frac{4 \pi v}{y^{2}}|Q(z, 1)|^{2}\right) .
$$

We next obtain (3.2) by showing that $-i y^{2 k} \frac{\partial}{\partial z} \Theta(z, \tau)$ equals

$$
\begin{array}{r}
-i v^{\frac{1}{2}} y^{2} \sum_{\substack{D \in \mathbb{Z} \\
Q \in \mathcal{Q}_{D}}} Q(z, 1)^{k-1} e^{-4 \pi Q_{z}^{2} v} e^{2 \pi i D \tau}\left(k \frac{\partial}{\partial z}\left(\frac{Q(z, 1)}{y^{2}}\right)-\frac{8 \pi}{y^{2}} Q_{z} Q(z, 1) v \frac{\partial}{\partial z} Q_{z}\right) \\
=v^{\frac{1}{2}} \sum_{\substack{D \in \mathbb{Z} \\
Q \in \mathcal{Q}_{D}}} Q_{z} Q(z, 1)^{k-1} e^{-4 \pi Q_{z}^{2} v} e^{2 \pi i D \tau}\left(k-\frac{4 \pi v}{y^{2}}|Q(z, 1)|^{2}\right)
\end{array}
$$

where in the last line we have used

$$
y^{2} \frac{\partial}{\partial z} Q_{z}=\frac{i}{2} Q(\bar{z}, 1) \quad \text { and } \quad y^{2} \frac{\partial}{\partial z}\left(y^{-2} Q(z, 1)\right)=i Q_{z}
$$

The following lemma relates the regularized inner products in positive and negative weights through the $\xi$-operator. 
Lemma 3.4. Suppose that $D>0$ is a discriminant and $z \notin E_{D}$. Then for every $s$ with $\operatorname{Re}(s) \geq \frac{k}{2}+\frac{1}{4}$ one has

$$
\left\langle\xi_{k+\frac{1}{2}}\left(P_{k+\frac{1}{2}, s, D}\right), \Theta^{*}(-\bar{z}, \cdot)\right\rangle^{\mathrm{reg}}=-\overline{\left\langle P_{k+\frac{1}{2}, s, D}, \xi_{\frac{3}{2}-k}\left(\Theta^{*}(-\bar{z}, \cdot)\right)\right\rangle^{\mathrm{reg}}}
$$

and

$$
\left\langle\xi_{\frac{3}{2}-k}\left(P_{\frac{3}{2}-k, s, D}\right), \Theta(z, \cdot)\right\rangle^{\mathrm{reg}}=-\overline{\left\langle P_{\frac{3}{2}-k, s, D}, \xi_{k+\frac{1}{2}}(\Theta(z, \cdot))\right\rangle^{\mathrm{reg}}} .
$$

Proof. Note that all of the regularized integrals exist, as will be shown in the proofs of Theorems $1.1(2)$ and $1.3(2)$. We begin with the proof of (3.5) and abbreviate $P:=P_{k+\frac{1}{2}, s, D}$. By Lemma 2.1, we have

$$
\begin{aligned}
& \left\langle\xi_{k+\frac{1}{2}}(P), \Theta^{*}(-\bar{z}, \cdot)\right\rangle^{\mathrm{reg}}+\overline{\left\langle P, \xi_{\frac{3}{2}-k}\left(\Theta^{*}(-\bar{z}, \cdot)\right)\right\rangle^{\mathrm{reg}}} \\
& \quad=-\frac{1}{6} \lim _{T \rightarrow \infty} \int_{\partial \mathcal{F}_{T}(4)} \overline{P(\tau) \Theta^{*}(-\bar{z}, \tau)} d \bar{\tau}
\end{aligned}
$$

provided that the limit exists. Hence, our goal is to show that the limit on the righthand side is zero. A standard argument reduces this claim to showing that

$$
\lim _{T \rightarrow \infty} \int_{0}^{1} P(u+i T) \Theta^{*}(-\bar{z}, u+i T) d u=0
$$

as well as vanishing of similar integrals around the other cusps of $\Gamma_{0}(4)$. However, since both $P$ and $\Theta^{*}$ are in Kohnen's plus space, the vanishing of the corresponding integrals at the other cusps may be reduced to showing that (3.7) vanishes.

In order to prove (3.7), we first recall the growth condition (2.11) and note that $\Theta^{*}(-\bar{z}, u+i T)$ decays exponentially as $T \rightarrow \infty$. Indeed, using (3.3), one can show that for fixed $z \in \mathbb{H}$ the quadratic form

$$
Q^{*}(a, b, c):=D-\frac{2|Q(z, 1)|^{2}}{y^{2}}=-D+2 Q_{z}^{2}
$$

is positive definite on the lattice of all binary quadratic forms $Q=[a, b, c] \in \mathcal{Q}_{D}$. After evaluating the integral over $u$, one reduces (3.7) to showing that

$$
\lim _{T \rightarrow \infty} R_{T}=0
$$

where

$$
R_{T}:=\mathcal{M}_{k+\frac{1}{2}, s}(4 \pi D T) T^{k} \sum_{Q \in \mathcal{Q}_{D}} Q_{-\bar{z}} Q(-\bar{z}, 1)^{k-1} e^{-\frac{4 \pi|Q(-\bar{z}, 1)|^{2} T}{y^{2}}} e^{2 \pi D T} .
$$

However, the asymptotic behavior for the Whittaker function coming from (2.8) and (2.9) yields

$$
\mathcal{M}_{k+\frac{1}{2}, s}(4 \pi D T) \ll_{k, s, D} e^{2 \pi D T} T^{-k-\frac{1}{2}} .
$$

Using (3.3), we may hence bound

$$
R_{T} \ll_{k, s, D} T^{-\frac{1}{2}} \sum_{Q \in \mathcal{Q}_{D}} Q_{-\bar{z}} Q(-\bar{z}, 1)^{k-1} e^{-4 \pi Q_{-\bar{z}}^{2} T} .
$$

Since $z \notin E_{D}$ (and hence $\left.-\bar{z} \notin E_{D}\right), Q_{-\bar{z}}^{2}>0$ for every $Q \in \mathcal{Q}_{D}$ and hence $R_{T}$ exhibits exponential decay as $T \rightarrow \infty$. This concludes (3.7), yielding (3.5). The proof of (3.6) follows analogously. 


\section{Image of the theta lift $\Phi_{k}$}

In this section, we introduce a spectral parameter in the classical Shintani lift.

Proof of Theorem 1.1 (2). In order to compute the regularized inner product, we use a method of Zagier [38]. He defined a regularization that he used for functions which grow at most polynomially, but the method may be extended to the functions of interest here, as we now describe. We first define

$$
\mathbb{H}_{T}:=\bigcup_{\gamma \in \mathrm{SL}_{2}(\mathbb{Z})} \gamma \mathcal{F}_{T}=\bigcup_{\gamma \in \Gamma_{0}(4)} \gamma \mathcal{F}_{T}(4) .
$$

We first use (2.5) together with the fact that $\Theta=\Theta \mid$ pr to compute

$$
\begin{aligned}
\left\langle P_{k+\frac{1}{2}, s, D}, \Theta(z, \cdot)\right\rangle^{\mathrm{reg}} & =\left\langle P_{k+\frac{1}{2}, s, \Gamma_{0}(4), D} \mid \mathrm{pr}, \Theta(z, \cdot)\right\rangle^{\mathrm{reg}} \\
& =\left\langle P_{k+\frac{1}{2}, s, \Gamma_{0}(4), D}, \Theta(z, \cdot)\right\rangle^{\mathrm{reg}} .
\end{aligned}
$$

Then the usual unfolding argument yields

$$
\left\langle P_{k+\frac{1}{2}, s, D}, \Theta(z, \cdot)\right\rangle^{\mathrm{reg}}=\frac{1}{6} \lim _{T \rightarrow \infty} \int_{\Gamma_{\infty} \backslash \mathbb{H}_{T}} \psi_{D, k+\frac{1}{2}}(s ; \tau) \overline{\Theta(z, \tau)} v^{k+\frac{1}{2}} \frac{d u d v}{v^{2}} .
$$

We now rewrite

$$
\mathbb{H}_{T}=\{\tau \in \mathbb{H} \mid \operatorname{Im}(\tau) \leq T\} \backslash \bigcup_{\substack{c \geq 1 \\ a \in \mathbb{Z} \\(a, c)=1}} S_{\frac{a}{c}}(T)
$$

where $S_{\frac{a}{c}}(T)$ is the disc of radius $\frac{1}{2 c^{2} T}$ tangent to the real axis at $\frac{a}{c}$. Hence, we have

$$
\left\langle P_{k+\frac{1}{2}, s, D}, \Theta(z, \cdot)\right\rangle^{\mathrm{reg}}=\lim _{T \rightarrow \infty}\left(I_{1}(T)+I_{2}(T)\right)
$$

where

$$
\begin{aligned}
& I_{1}(T):=\frac{1}{6} \int_{0}^{T} \int_{0}^{1} \psi_{D, k+\frac{1}{2}}(s ; \tau) \overline{\Theta(z, \tau)} v^{k+\frac{1}{2}} \frac{d u d v}{v^{2}} \\
& I_{2}(T):=-\frac{1}{6} \sum_{c \geq 1} \sum_{\substack{a(\bmod c) \\
(a, c)=1}} \int_{S \frac{a}{c}(T)} \psi_{D, k+\frac{1}{2}}(s ; \tau) \overline{\Theta(z, \tau)} v^{k+\frac{1}{2}} \frac{d u d v}{v^{2}} .
\end{aligned}
$$

We first consider $I_{1}(T)$. Evaluating the integral over $u$ and using (3.3), we obtain

$$
\begin{aligned}
\lim _{T \rightarrow \infty} I_{1}(T) & =\frac{(4 \pi D)^{\frac{k}{2}+\frac{1}{4}}}{6 y^{2 k} \Gamma(2 s)} \sum_{Q \in Q_{D}} Q(\bar{z}, 1)^{k} \int_{0}^{\infty} \mathcal{M}_{k+\frac{1}{2}, s}(4 \pi D v) e^{-2 \pi D v-4 \pi Q_{z}^{2} v} v^{k-1} d v \\
& =\frac{(4 \pi D)^{\frac{1}{4}-\frac{k}{2}}}{6 y^{2 k} \Gamma(2 s)} \sum_{Q \in \mathcal{Q}_{D}} Q(\bar{z}, 1)^{k} I\left(\frac{D y^{2}}{|Q(\tau, 1)|^{2}}\right)
\end{aligned}
$$

where for $0<w<1$ we define

$$
I(w):=\int_{0}^{\infty} \mathcal{M}_{k+\frac{1}{2}, s}(v) e^{\frac{v}{2}} e^{-v w^{-1}} v^{k-1} d v
$$


In the case that $s \neq \frac{k}{2}+\frac{1}{4}$, we insert the definition (2.6) of $\mathcal{M}_{k+\frac{1}{2}, s}(v)$ and then substitute the integral representation (2.7) of the $M$-Whittaker function when $\operatorname{Re}(s)>$ $\frac{k}{2}$. The change of variables $t \rightarrow 1-t$ yields that $I(w)$ equals

$$
\begin{gathered}
\frac{\Gamma(2 s)}{\Gamma\left(s+\frac{k}{2}+\frac{1}{4}\right) \Gamma\left(s-\frac{k}{2}-\frac{1}{4}\right)} \int_{0}^{1}(1-t)^{s+\frac{k}{2}-\frac{3}{4}} t^{s-\frac{k}{2}-\frac{5}{4}} \int_{0}^{\infty} v^{s+\frac{k}{2}-\frac{5}{4}} e^{-v\left(w^{-1}-t\right)} d v d t \\
=\frac{\Gamma(2 s) \Gamma\left(s+\frac{k}{2}-\frac{1}{4}\right) w^{s+\frac{k}{2}-\frac{1}{4}}}{\Gamma\left(s+\frac{k}{2}+\frac{1}{4}\right) \Gamma\left(s-\frac{k}{2}-\frac{1}{4}\right)} \int_{0}^{1}(1-t)^{s+\frac{k}{2}-\frac{3}{4}} t^{s-\frac{k}{2}-\frac{5}{4}}(1-w t)^{-\frac{k}{2}-s+\frac{1}{4}} d t .
\end{gathered}
$$

We then rewrite the integral using the Euler integral representation for ${ }_{2} F_{1}$ (see (15.3.1) in [1]), given for $\operatorname{Re}(C)>\operatorname{Re}(B)>0$ and $|w|<1$ by

$$
{ }_{2} F_{1}(A, B ; C ; w)=\frac{\Gamma(C)}{\Gamma(B) \Gamma(C-B)} \int_{0}^{1} t^{B-1}(1-t)^{C-B-1}(1-w t)^{-A} d t .
$$

Thus

$$
I(w)=\Gamma\left(s+\frac{k}{2}-\frac{1}{4}\right) w^{s+\frac{k}{2}-\frac{1}{4}}{ }_{2} F_{1}\left(s+\frac{k}{2}-\frac{1}{4}, s-\frac{k}{2}-\frac{1}{4} ; 2 s ; w\right) .
$$

Inserting this into (4.2) shows that $\lim _{T \rightarrow \infty} I_{1}(T)=f_{k, s, D}$.

For $s=\frac{k}{2}+\frac{1}{4}$, inserting (2.8) into (4.2) yields

$$
\lim _{T \rightarrow \infty} I_{1}(T)=\frac{D^{\frac{k}{2}+\frac{1}{4}} \Gamma(k)}{6(4 \pi)^{\frac{k}{2}-\frac{1}{4}} \Gamma\left(k+\frac{1}{2}\right)} \sum_{Q \in \mathcal{Q}_{D}} Q(z, 1)^{-k}=f_{k, \frac{k}{2}+\frac{1}{4}, D}(z) .
$$

To conclude (1.8), it remains to show that $I_{2}(T)$ vanishes as $T \rightarrow \infty$. We first assume that $4 \mid c$ and choose $\gamma=\left(\begin{array}{ll}a & b \\ c & d\end{array}\right) \in \Gamma_{0}(4)$. A direct calculation shows that

$$
\gamma S_{\frac{a}{c}}=\{\tau \in \mathbb{H} \mid v \geq T\} .
$$

Hence, the change of variables $\tau \rightarrow \gamma \tau$, together with the modularity of $\Theta$ in Proposition 3.2 , yields

$$
I_{2}(T)=-\frac{1}{6} \int_{T}^{\infty} \int_{-\infty}^{\infty} \overline{\Theta(-\bar{z}, \tau)}(c \bar{\tau}+d)^{k+\frac{1}{2}} \psi_{D, k+\frac{1}{2}}(s ; \gamma \tau) \operatorname{Im}(\gamma \tau)^{k+\frac{1}{2}} \frac{d u d v}{v^{2}}
$$

Using the facts that $\operatorname{Im}(\gamma \tau)=\frac{v}{|c \tau+d|^{2}}$ and $\Theta$ is translation invariant, the integral may be rewritten as

$$
-\left.\frac{1}{6} \int_{T}^{\infty} \int_{0}^{1} \overline{\Theta(-\bar{z}, \tau)} \sum_{n=-\infty}^{\infty} \psi_{D, k+\frac{1}{2}}(s ; \tau)\right|_{k+\frac{1}{2}} \gamma\left(\begin{array}{cc}
1 & n \\
0 & 1
\end{array}\right) v^{k+\frac{1}{2}} \frac{d u d v}{v^{2}} .
$$

Taking the sum over all $a, c$ with $4 \mid c>0$, the inner sum precisely evaluates as

$$
P_{k+\frac{1}{2}, s, \Gamma_{0}(4), D}-\psi_{D, k+\frac{1}{2}}(s ; \tau) .
$$

Comparing the polynomial growth in $(2.11)$ with the exponential decay of $\Theta(-\bar{z}, \tau)$ towards $i \infty$, one concludes that the limit $T \rightarrow \infty$ vanishes. A similar argument shows that the contribution to $I_{2}(T)$ coming from $4 \nmid c$ also vanishes as $T \rightarrow \infty$. This yields (1.8). 


\section{Image of the theta lift $\Phi_{1-k}^{*}$}

We next compute the image of $\Phi_{1-k}^{*}$ with the method from Section 4 .

Proof of Theorem 1.3 (2). Following the argument in the proof of Theorem $1.1(2)$, we may reduce the theorem to evaluating

$$
\begin{aligned}
\frac{1}{6} \lim _{T \rightarrow \infty} \int_{0}^{T} \int_{0}^{1} \psi_{D, \frac{3}{2}-k}(s ; \tau) \overline{\Theta^{*}(-\bar{z}, \tau)} v^{\frac{3}{2}-k} \frac{d u d v}{v^{2}} \\
\quad-\frac{1}{6} \lim _{T \rightarrow \infty} \sum_{c \geq 1} \sum_{\substack{(\bmod c) \\
(a, c)=1}} \int_{S_{\frac{a}{c}}(T)} \psi_{D, \frac{3}{2}-k}(s ; \tau) \overline{\Theta^{*}(-\bar{z}, \tau)} v^{\frac{3}{2}-k} \frac{d u d v}{v^{2}} .
\end{aligned}
$$

Using the argument from before, the second summand vanishes. We use (3.3) to rewrite the exponential in the theta series as

$$
\left(b^{2}-4 a c\right) u+i v\left(2 Q_{-\bar{z}}^{2}+\left(b^{2}-4 a c\right)\right) .
$$

Therefore, evaluating the integral over $u$ and then making the change of variables $Q \rightarrow \widetilde{Q}$ (as defined before (3.4)), it suffices to compute

$$
\frac{1}{6}(4 \pi D)^{\frac{1}{4}-\frac{k}{2}} \Gamma(2 s)^{-1} \sum_{Q \in \mathcal{Q}_{D}} Q_{z} Q(z, 1)^{k-1} \mathcal{I}\left(\frac{D y^{2}}{|Q(z, 1)|^{2}}\right),
$$

where

$$
\mathcal{I}(w):=\int_{0}^{\infty} \mathcal{M}_{\frac{3}{2}-k, s}(-v) e^{\frac{v}{2}} v^{-\frac{1}{2}} e^{-v w^{-1}} d v
$$

Inserting the definition (2.6) of $\mathcal{M}_{\frac{3}{2}-k, s}$ and the integral representation (2.7) of the $M$-Whittaker function, we evaluate

$$
\begin{aligned}
\mathcal{I}(w)= & \frac{\Gamma(2 s)}{\Gamma\left(s-\frac{k}{2}+\frac{3}{4}\right) \Gamma\left(s+\frac{k}{2}-\frac{3}{4}\right)} \\
& \times \int_{0}^{1} t^{s+\frac{k}{2}-\frac{7}{4}}(1-t)^{s-\frac{k}{2}-\frac{1}{4}} \int_{0}^{\infty} v^{s+\frac{k}{2}-\frac{5}{4}} e^{-v\left(t-1+w^{-1}\right)} d v d t \\
= & \frac{\Gamma(2 s) \Gamma\left(s+\frac{k}{2}-\frac{1}{4}\right)}{\Gamma\left(s-\frac{k}{2}+\frac{3}{4}\right) \Gamma\left(s+\frac{k}{2}-\frac{3}{4}\right)} \\
& \times w^{s+\frac{k}{2}-\frac{1}{4}} \int_{0}^{1}(1-t)^{s+\frac{k}{2}-\frac{7}{4}} t^{s-\frac{k}{2}-\frac{1}{4}}(1-w t)^{-s-\frac{k}{2}+\frac{1}{4}} d t .
\end{aligned}
$$

We again employ the Euler integral representation (4.3) to show that

$$
\mathcal{I}(w)=\Gamma\left(s+\frac{k}{2}-\frac{1}{4}\right) w^{s+\frac{k}{2}-\frac{1}{4}}{ }_{2} F_{1}\left(s+\frac{k}{2}-\frac{1}{4}, s-\frac{k}{2}+\frac{3}{4} ; 2 s ; w\right) .
$$

We then rewrite the hypergeometric function by using the Euler transform

$$
{ }_{2} F_{1}(A, B ; C ; w)=(1-w)^{C-A-B}{ }_{2} F_{1}(C-A, C-B ; C ; w)
$$

to yield

$$
\mathcal{I}(w)=\Gamma\left(s+\frac{k}{2}-\frac{1}{4}\right)(1-w)^{-\frac{1}{2}} w^{s+\frac{k}{2}-\frac{1}{4}}{ }_{2} F_{1}\left(s-\frac{k}{2}+\frac{1}{4}, s+\frac{k}{2}-\frac{3}{4} ; 2 s ; w\right) .
$$


Finally, we conclude that (5.2) equals (1.8) by using (3.3) to rewrite $\left|Q_{z}\right|$ in terms of $\frac{D y^{2}}{|Q(z, 1)|^{2}}$.

We next establish the injectivity of the lift.

Proof of Theorem 1.4 (2). Since the Poincaré series $P_{\frac{3}{2}-k, s, D}$ span the space of weak Maass forms and are linearly independent (which can be seen by comparing their principal parts), it is enough to show that the functions $\mathcal{F}_{1-k, s, D}$ are linearly independent. This follows by proving that any linear combination

$$
\mathcal{F}:=\sum_{j=1}^{n} a_{j} \mathcal{F}_{1-k, s, D_{j}}
$$

with $a_{j}$ not all zero exhibits discontinuities and is hence non-zero. Comparing the sets $E_{D_{j}}$ of geodesics defined in (1.4) implies the result.

\section{Relation between positive and negative weight local Maass forms}

In this section, we relate $f_{k, s, D}$ and $\mathcal{F}_{1-k, s, D}$.

Proof of Theorem 1.5. We prove (1.10) by establishing that for $P:=P_{\frac{3}{2}-k, s, D}$ and $z \notin E_{D}$, one has

$$
\xi_{2-2 k}\left(\Phi_{1-k}^{*}(P)(z)\right)=2 \Phi_{k}\left(\xi_{\frac{3}{2}-k}(P)\right)(z) .
$$

We first use (3.6) and then (3.1) to obtain for $z \notin E_{D}$

$$
\begin{aligned}
\Phi_{k}\left(\xi_{\frac{3}{2}-k}(P)\right)(z) & =\left\langle\xi_{\frac{3}{2}-k}(P), \Theta(z, \cdot)\right\rangle^{\mathrm{reg}}=-\overline{\left\langle P, \xi_{k+\frac{1}{2}}(\Theta(z, \cdot))\right\rangle^{\mathrm{reg}}} \\
& \left.=-\left\langle P,-i y^{2-2 k} \frac{\partial}{\partial z} \Theta^{*}(-\bar{z}, \cdot)\right\rangle\right\rangle^{\mathrm{reg}} \\
& =\frac{i y^{2-2 k}}{6} \frac{\partial}{\partial z} \int_{\mathcal{F}_{0}(4)}^{\mathrm{reg}} \overline{P(\tau)} \Theta^{*}(-\bar{z}, \tau) v^{\frac{3}{2}-k} \frac{d u d v}{v^{2}} \\
& =i y^{2-2 k} \frac{\partial}{\partial z} \overline{\left\langle P, \Theta^{*}(-\bar{z}, \cdot)\right\rangle^{\mathrm{reg}}}
\end{aligned}
$$

Since

$$
\xi_{\kappa}(G(z))=2 i y^{\kappa} \frac{\partial}{\partial z} \overline{G(z)}
$$

we conclude (6.1) from (6.2).

We now apply Theorems $1.3(2),(6.1),(2.13)$, and finally Theorem $1.1(2)$ to yield

$$
\begin{aligned}
\xi_{2-2 k}\left(\mathcal{F}_{1-k, s, D}(z)\right) & =\xi_{2-2 k}\left(\Phi_{1-k}^{*}\left(P_{\frac{3}{2}-k, s, D}\right)(z)\right)=2 \Phi_{k}\left(\xi_{\frac{3}{2}-k}\left(P_{\frac{3}{2}-k, s, D}\right)\right)(z) \\
& =2\left(\bar{s}-\frac{3}{4}+\frac{k}{2}\right) \Phi_{k}\left(P_{k+\frac{1}{2}, \bar{s}, D}\right)(z)=2\left(\bar{s}-\frac{3}{4}+\frac{k}{2}\right) f_{k, \bar{s}, D}(z) .
\end{aligned}
$$

This concludes the proof of (1.10).

We next prove (1.11). Denoting $P:=P_{k+\frac{1}{2}, s, D}$, we use (3.5) to conclude that for $z \notin E_{D}$

$$
\Phi_{1-k}^{*}\left(\xi_{k+\frac{1}{2}}(P)\right)(z)=\left\langle\xi_{k+\frac{1}{2}}(P), \Theta^{*}(-\bar{z}, \cdot)\right\rangle^{\mathrm{reg}}=-\overline{\left\langle P, \xi_{\frac{3}{2}-k}\left(\Theta^{*}(-\bar{z}, \cdot)\right)\right\rangle^{\mathrm{reg}}} .
$$


We then employ (3.2) and (6.3) to obtain

$$
\Phi_{1-k}^{*}\left(\xi_{k+\frac{1}{2}}(P)\right)(z)=i y^{2 k} \frac{\partial}{\partial z} \overline{\langle P, \Theta(z, \cdot)\rangle^{\mathrm{reg}}}=\frac{1}{2} \xi_{2 k}\left(\Phi_{k}(P)(z)\right) .
$$

Combining this with Theorem 1.3 (2), (2.13), and Theorem 1.1 (2) yields

$$
\begin{aligned}
\left(\bar{s}-\frac{k}{2}-\frac{1}{4}\right) \mathcal{F}_{1-k, \bar{s}, D}(z) & =\left(\bar{s}-\frac{k}{2}-\frac{1}{4}\right) \Phi_{1-k}^{*}\left(P_{\frac{3}{2}-k, \bar{s}, D}\right)(z) \\
& =\Phi_{1-k}^{*}\left(\xi_{k+\frac{1}{2}}\left(P_{k+\frac{1}{2}, s, D}\right)\right)(z)=\frac{1}{2} \xi_{2 k}\left(\Phi_{k}\left(P_{k+\frac{1}{2}, s, D}\right)(z)\right) \\
& =\frac{1}{2} \xi_{2 k}\left(f_{k, s, D}(z)\right) .
\end{aligned}
$$

We are now ready to prove Theorems 1.1 (1) and 1.3 (1).

Proof of Theorem 1.1 (1). Note that

$$
\overline{\Theta(z, \tau)}=\Theta(-\bar{z},-\bar{\tau}) .
$$

Hence $f_{k, s, D}$ is modular of weight $2 k$ by Proposition 3.2 .

The functions $f_{k, s, D}$ are continuous since for $\operatorname{Re}(C)>\operatorname{Re}(A+B)$, the hypergeometric function ${ }_{2} F_{1}(A, B ; C ; w)$, and hence $\varphi_{s}(w)$, is continuous for $w \leq 1$. This implies condition (3).

For $z \notin E_{D},(1.11)$ and (1.10) imply that

$$
\Delta_{2 k}\left(f_{k, s, D}(z)\right)=-\xi_{2-2 k}\left(\xi_{2 k}\left(f_{k, s, D}(z)\right)\right)=4 \lambda_{s} f_{k, s, D}(z) .
$$

A straightforward calculation shows that $f_{k, s, D}(z)$ grows at most polynomially as $y \rightarrow \infty$.

Finally, one uses (4.4) and the duplication formula for the $\Gamma$-function to conclude (1.5).

Remark. The non-differentiability of $f_{k, s, D}$ follows by using (1.11) and then proving that the functions $\mathcal{F}_{1-k, s, D}$ are not continuous. Computational evidence indicates that $f_{k, s, D}(z)$ decays exponentially as $y \rightarrow \infty$. Even in the special case that $s=\frac{k}{2}+\frac{1}{4}$, a naive termwise bound on $f_{k, D}$ in (1.1) only yields polynomial decay as $y \rightarrow \infty$. To prove exponential decay, one would need to compute the Fourier-type expansions in the connected components of $\mathbb{H} \backslash E_{D}$. Although there are standard methods to compute these expansions for theta lifts, we do not require them for the purposes of this paper.

Proof of Theorem 1.3 (1). Noting that

$$
\overline{\Theta^{*}(-\bar{z}, \tau)}=\Theta^{*}(z,-\bar{\tau}),
$$

Proposition 3.2 implies that $\mathcal{F}_{1-k, s, D}$ is modular of weight $2-2 k$.

The proof that $\mathcal{F}_{1-k, s, D}$ is an eigenfunction under $\Delta_{2-2 k}$ with eigenvalue $4 \lambda_{s}$ follows by (1.10) and (1.11) precisely as in the proof of Theorem 1.1 (1).

In order to show condition (3) in the definition of local Maass forms, we first note that $\varphi_{s}^{*}(w)$ is continuous for $0<w \leq 1$. The locally uniform convergence of the sum allows us to pull the limit $r \rightarrow 0^{+}$of $\mathcal{F}_{1-k, s, D}(z \pm i r)$ into each term. Define

$$
\mathscr{B}_{z}:=\left\{Q \in \mathcal{Q}_{D} \mid Q_{z}=0\right\} \text {. }
$$


By Lemma 5.1 of [7], there are only finitely many $Q \in \mathscr{B}_{z}$. Note that

$$
\operatorname{sgn}\left(Q_{z}\right)=\operatorname{sgn}\left(Q_{z \pm i r}\right)
$$

for $r$ sufficiently small and $Q \notin \mathscr{B}_{z}$, while for $Q \in \mathscr{B}_{z}$ one has

$$
\operatorname{sgn}\left(Q_{z+i r}\right)=-\operatorname{sgn}\left(Q_{z-i r}\right) .
$$

Hence, since the terms of $\mathcal{F}_{1-k, s, D}(z)$ with $Q \in \mathscr{B}_{z}$ vanish,

$$
\begin{aligned}
\frac{1}{2} & \lim _{r \rightarrow 0^{+}}\left(\mathcal{F}_{1-k, s, D}(z+i r)+\mathcal{F}_{1-k, s, D}(z-i r)\right) \\
\quad= & \sum_{Q \notin \mathscr{B}_{z}} \operatorname{sgn}\left(Q_{z}\right) Q(z, 1)^{k-1} \varphi_{s}^{*}\left(\frac{D y^{2}}{|Q(z, 1)|^{2}}\right)=\mathcal{F}_{1-k, s, D}(z) .
\end{aligned}
$$

A direct calculation shows that $\mathcal{F}_{1-k, s, D}(z)$ grows at most polynomially as $y \rightarrow \infty$.

Remark. To show that $\mathcal{F}_{1-k, s, D}$ exhibits discontinuities along the set $E_{D}$, one computes

$$
\lim _{r \rightarrow 0^{+}}\left(\mathcal{F}_{1-k, s, D}(z+i r)-\mathcal{F}_{1-k, s, D}(z-i r)\right)
$$

similarly as in the proof of Theorem 1.3 (1). It is shown to be non-zero by using Gauss's summation formula to conclude that $\varphi_{s}^{*}(1) \neq 0$.

If $D$ is not a square and $\operatorname{Re}(s) \geq \frac{k}{2}+\frac{1}{4}$, then computational evidence indicates that $\mathcal{F}_{1-k, s, D}$ is bounded as $y \rightarrow \infty$.

\section{Hecke operators}

In this section, we consider the action of the Hecke operators on the theta lifts.

Proof of Theorem 1.4 (1). Since the Poincaré series span the space of weight $\frac{3}{2}-k$ weak Maass forms, it suffices to compute the action of the Hecke operators on Poincaré series. As in the proof of Theorem 1.5 of [7], one can show that

$$
\left.\mathcal{F}_{1-k, s, D}\right|_{2-2 k} T_{p}=\mathcal{F}_{1-k, s, D p^{2}}+p^{-k}\left(\frac{D}{p}\right) \mathcal{F}_{1-k, s, D}+p^{1-2 k} \mathcal{F}_{1-k, s, \frac{D}{p^{2}}} .
$$

Hence by Theorem 1.3 (1), equation (1.9) follows by the easily verified identity

$$
\left.P_{\frac{3}{2}-k, s, D}\right|_{\frac{3}{2}-k} T_{p^{2}}=P_{\frac{3}{2}-k, s, D p^{2}}+p^{-k}\left(\frac{D}{p}\right) P_{\frac{3}{2}-k, s, D}+p^{1-2 k} P_{\frac{3}{2}-k, s, \frac{D}{p^{2}}} .
$$

We now move on to the positive weight case.

Proof of Theorem 1.2. We first prove Theorem $1.2(1)$. Let $\mathcal{H}$ be a weight $2 k$ local Maass form with exceptional set $E_{D}$, which is continuous everywhere. Since continuity is preserved by the Hecke operators, one easily checks that $\left.\mathcal{H}\right|_{2 k} T_{p}$ is a local Maass form. To determine the exceptional set for $\mathcal{H}$, recall that the weight $2 k$ Hecke operator may be written as

$$
\left.\mathcal{H}\right|_{2 k} T_{p}(\tau)=p^{2 k-1} \mathcal{H}(p \tau)+p^{-1} \sum_{r} \mathcal{H}\left(\frac{\tau+r}{p}\right)
$$


By computing the image of $E_{D}$ under $\tau \rightarrow p \tau$ and $\tau \rightarrow \frac{\tau+r}{p}$, one concludes that $\left.\mathcal{H}\right|_{2 k} T_{p}$ has exceptional set $E:=E_{D p^{2}} \supset E_{D}$. Hence it suffices to prove the statement for $z \notin E$.

Suppose that $H$ is a weak Maass form of weight $k+\frac{1}{2}$ with eigenvalue $\lambda_{s}$ under $\Delta_{k+\frac{1}{2}}$. Since $\xi_{\frac{3}{2}-k}$ surjects onto the space of weak Maass forms of weight $k+\frac{1}{2}$ with eigenvalue $\lambda_{s}$ (see [13]), we may choose such a weight $\frac{3}{2}-k$ weak Maass form $G$ such that $\xi_{\frac{3}{2}-k}(G)=H$. However then by (1.9) and (6.1), and the fact that the Hecke operators commute with $\xi_{2-2 k}$, for $z \notin E$, we have that

$$
\left.\Phi_{k}(H)\right|_{2 k} T_{p}(z)=\left.\frac{1}{2} \xi_{2-2 k}\left(\Phi_{1-k}^{*}(G)\right)\right|_{2 k} T_{p}(z)=\frac{1}{2} \xi_{2-2 k}\left(\Phi_{1-k}^{*}\left(\left.G\right|_{\frac{3}{2}-k} T_{p^{2}}\right)(z)\right) .
$$

We now use (6.5) and the fact that $\xi_{\frac{3}{2}-k}$ commutes with the Hecke operators to obtain

$$
\begin{aligned}
\xi_{2-2 k}\left(\Phi_{1-k}^{*}\left(\left.G\right|_{\frac{3}{2}-k} T_{p^{2}}\right)(z)\right) & =2 \Phi_{k}\left(\xi_{\frac{3}{2}-k}\left(\left.G\right|_{\frac{3}{2}-k} T_{p^{2}}\right)\right)(z) \\
& =2 \Phi_{k}\left(\left.H\right|_{k+\frac{1}{2}} T_{p^{2}}\right)(z),
\end{aligned}
$$

as desired.

We move on to Theorem $1.2(2)$. Assume that $\Phi_{k}(F) \equiv 0$ for a weak Maass form $F$ with eigenvalue $\lambda_{s} \neq 0$. Writing $G:=-\left(4 \lambda_{s}\right)^{-1} \xi_{k+\frac{1}{2}}(F)$, by (6.1) we have that

$$
0=\xi_{2-2 k}\left(\Phi_{1-k}^{*}(G)\right) \text {. }
$$

Since $\Phi_{1-k}^{*}(G)$ is an eigenfunction under $\Delta_{2-2 k}$ with eigenvalue $4 \lambda_{s} \neq 0$, we have

$$
0=-\left(4 \lambda_{s}\right)^{-1} \xi_{2 k}\left(\xi_{2-2 k}\left(\Phi_{1-k}^{*}(G)\right)\right)=\Phi_{1-k}^{*}(G) .
$$

Since $\Phi_{1-k}^{*}$ is injective, we conclude that $G \equiv 0$. However,

$$
\xi_{\frac{3}{2}-k}(G)=F,
$$

and hence $F \equiv 0$.

\section{Acknowledgments}

The authors thank Jan Bruinier for suggesting to investigate the connection between $\mathcal{F}_{1-k, D}$ and theta lifts and for fruitful discussion. The authors also thank Jens Funke for helpful comments which aided the exposition. The research of the first author was supported by the Alfried Krupp Prize for Young University Teachers of the Krupp Foundation.

\section{References}

[1] M. Abramowitz and I. Stegun, Handbook of mathematical functions, Dover Publications, New York, 1964.

[2] G. Andrews, Partitions, Durfee symbols, and the Atkin-Garvan moments of ranks, Invent. Math. 169 (2007), 37-73.

[3] R. Borcherds, Automorphic forms with singularities on Grassmannians, Invent. Math. 132 (1998), 491-562.

[4] K. Bringmann, On the explicit construction of higher deformations of partition statistics, Duke Math. J. 144 (2008), 195-233.

[5] K. Bringmann, N. Diamantis and M. Raum, Mock period functions, sesquiharmonic Maass forms, and non-critical values of L-functions, Adv. Math. 233 (2013), 115-134.

[6] K. Bringmann, F. Garvan and K. Mahlburg, Partition statistics and quasiharmonic Maass forms, Int. Math. Res. Not. 2009 (2009), 63-97. 
[7] K. Bringmann, B. Kane and W. Kohnen, Locally harmonic Maass forms and rational period functions, submitted for publication.

[8] K. Bringmann, B. Kane and S. Zwegers, On a completed generating function of locally harmonic Maass forms, Compositio Math., accepted for publication.

[9] K. Bringmann and K. Ono, The $f(q)$ mock theta function conjecture and partition ranks, Invent. Math. 165 (2006), 243-266.

[10] - Arithmetic properties of coefficients of half-integral weight Maass-Poincaré series, Math. Ann. 337 (2007), 591-612.

[11] - Dyson's ranks and Maass forms, Ann. Math. 171 (2010), 419-449.

[12] J. Bruinier, Borcherds products on $O(2, l)$ and Chern classes of Heegner divisors, Lectures Notes in Mathematics, 1780, Springer, Berlin, 2002.

[13] J. Bruinier and J. Funke, On two geometric theta lifts, Duke Math. J. 125 (2004), 45-90.

[14] J. Bruinier and K. Ono, Heegner divisors, L-functions, and Maass forms, Ann. Math. 172 (2010), 2135-2181.

[15] J. Bruinier and T. Yang, Faltings heights of CM cycles and derivatives of L-functions, Invent. Math. 177 (2009), 631-681.

[16] T. Eguchi, H. Ooguri and Y. Tachikawa, Notes on the K3 surface and the Mathieu group $M_{24}$, Exp. Math. 20 (2011), 91-96.

[17] J. Fay, Fourier coefficients of the resolvent for a Fuchsian group, J. Reine Angew. Math. 293294 (1977), 143-203.

[18] V. Gritsenko and V. Nikulin, Automorphic forms and Lorentzian Kac-Moody algebras, Part II, Int. J. Math. 9 (1998), 201-275.

[19] J. Harvey and G. Moore, Algebras, BPS states, and strings, Nucl. Phys. B 463 (1996), 315-368.

[20] M. Hövel, Automorphe Formen mit Singularitäten auf dem hyperbolischen Raum, PhD thesis, Technical University of Darmstadt, 2012.

[21] R. Howe, $\theta$-series and invariant theory, in Automorphic forms, representations and $L$-functions, number XXXIII in Proc. Symp. Pure Mathematics, 275-285, Amer. Math. Soc., Providence, RI, 1979 .

[22] S. Katok and P. Sarnak, Heegner points, cycles, and Maass forms, Israel J. Math. 84 (1984), $193-227$.

[23] W. Kohnen, Modular forms of half-integral weight on $\Gamma_{0}(4)$, Math. Ann. 248 (1980), 249-266.

[24] W. Kohnen and D. Zagier, Values of L-series of modular forms at the center of the critical strip, Invent. Math. 64 (1981), 175-198.

[25] S. Niwa, Modular forms of half integral weight and the integral of certain theta-functions, Nagoya Math. J. 56 (1974), 147-161.

[26] T. Oda, On modular forms associated with indefinite quadratic forms of signature $(2, n-2)$, Math. Ann. 231 (1977), 97-144.

[27] H. Petersson, Einheitliche Begründung der Vollständigkeitssätze für die Poincaréschen Reihen von reeller Dimension bei beliebigen Grenzkreisgruppen von erster Art, Abh. Math. Semin. Univ. Hambg. 14 (1941), 22-60.

[28] S. Rallis and G. Schiffmann, Automorphic cusp forms constructed from the Weil representation, Bull. Amer. Math. Soc. 83 (1977), 271-275.

[29] — Discrete spectrum of the Weil representation, Bull. Amer. Math. Soc. 83 (1977) 267270.

[30] - Représentations supercuspidales du groupe métaplectique, C. R. Acad. Sci. Paris Sér. A-B 284 (1977), 931-934.

[31] - Automorphic forms constructed from the Weil representation: holomorphic case, Amer. J. Math. 100 (1978), 1049-1122.

[32] G. Shimura, On modular forms of half integral weight, Ann. Math. 97 (1973), 440-481.

[33] T. Shintani, On construction of holomorphic cusp forms of half integral weight, Nagoya Math. J. 58 (1975), 83-126.

[34] J. Tunnell, A classical Diophantine problem and modular forms of weight $\frac{3}{2}$, Invent. Math. 72 (1983), 323-334.

[35] M.-F. Vignéras, Série theta des formes quadratiques indéfinies, in Modular Functions of One Variable VI, (J.-P. Serre, D. Zagier, eds.), Lecture Notes in Mathematics, 227-239, 627, University of Bonn, Springer, Berlin, 1977. 
[36] J.-L. Waldspurger, Sur les coefficients de Fourier des formes modulaires de poids demi-entier, J. Math. Pures Appl. 60 (1981), 375-484.

[37] D. Zagier, Modular forms associated to real quadratic fields, Invent. Math. 30 (1975), 1-46.

[38] — The Rankin-Selberg method for automorphic functions which are not of rapid decay, J. Fac. Sci. Tokyo 28 (1982), 415-438.

[39] - Traces of singular moduli, in Motives, Polylogarithms and Hodge Theory Part I, (F. Bogomolov, L. Katzarkov, eds.), Lecture Notes in Mathematics, 211-244, International Press, 2002.

[40] S. Zwegers, Mock theta functions, PhD thesis, Utrecht University, 2002.

Mathematical Institute, University of Cologne, Weyertal 86-90, 50931 Cologne, GERMANY

E-mail address: kbringma@math.uni-koeln.de

Mathematical Institute, University of Cologne, Weyertal 86-90, 50931 Cologne, GerMANY

E-mail address: bkane@math.uni-koeln.de

University of Cologne, Weyertal 86-90, 50931 Cologne, Germany

E-mail address: mviazovs@math.uni-koeln.de 